\title{
MEJORA DE LA ATENCIÓN POST-EJERCICIO EN
}

ESCOLARES DEPORTISTAS

\section{IMPROVEMENT OF POST-EXERCISE ATTENTION IN SPORTS SCHOOLS}

\author{
Honorato Morente Oria ${ }^{1}$ \\ ${ }^{1}$ Facultad de Ciencias de la Educación, Universidad de Málaga (España)
}

Detalles del artículo:

Número de palabras: 2.669; Tablas: 0; Figuras: 0; Referencias:18

Recibido: ; Aceptado: ; Publicado: enero 2019

Conflicto de interés: El autor declara que no existen conflictos de interés.

Correspondencia del autor: Honorato Morente Oria, hmorente@uma.es

\section{Resumen}

El presente trabajo pretende analizar el desarrollo evolutivo de la atención y obtener datos normativos de la prueba de atención selectiva $\mathrm{d} 2$ en escolares que practican deporte y que estos se asemejen a resultados obtenidos en estudios previos. Participaron 14 escolares (8 chicas) con una edad comprendida entre 10 a 12 años (11.32 \pm .63 años). Todos los participantes tenían un mínimo de experiencia competitiva y se realizaron las pruebas el mismo día después de un entrenamiento de intensidad media. Los resultados de las pruebas muestran una mejora en los resultados de atención en el postest aunque estas diferencias no son estadísticamente significativas, así mismo no encontramos diferencias significativas en el nivel de atención en cuanto a género aunque los resultados de las chicas son ligeramente superiores a los chicos que podría ser debido al nivel madurativo de ambos sexos en esta etapa evolutiva.

Palabras claves: $\mathrm{d} 2$ test, training, karatecas.

\section{Abstract}

This paper aims to analyze the evolutionary development of attention and obtain normative data of the $\mathrm{d} 2$ care test in schoolchildren athletes and that these resemble results obtained in previous studies. 14 scholars ( 8 girls) with an age between 10 and 12 years (11.32 \pm .63 years) participated. All the participants had a minimum of competitive experience and the tests were done the same day after a medium intensity training. The results of the tests show an improvement in the results of attention in the posttest although these differences are not 
statistically significant, likewise we do not find significant differences in the level of attention regarding gender although the results of the girls are slightly higher than the guys that could be due to the maturational level of both sexes in this evolutionary stage.

Key words: attention, d2 test, training, athletes.

\section{INTRODUCCIÓN}

La atención ha sido definida como el mecanismo implicado directamente en la activación y el funcionamiento de los procesos de selección, distribución y mantenimiento de la actividad psicológica. Se trata, por tanto, de un proceso complejo que al estar constituida por distintos subprocesos ha dado lugar a establecer distintas clasificaciones y subtipos asociados también a diferentes mecanismos o circuitos neurales (Cuesta et al., 2007).

Las alteraciones atencionales están presentes en determinados trastornos como la negligencia unilateral, el síndrome de Balint y la prosopagnosia o ceguera facial (Castillo, 2009). Asimismo, las alteraciones atencionales están asociadas a diferentes enfermedades como las demencias, epilepsia y a trastornos del desarrollo como el autismo o el trastorno por déficit de atención con o sin hiperactividad (TDAH) (Miranda, García y Soriano, 2005).

En el trabajo que aquí presentamos nos centramos en la atención considerada ésta como una función independiente y diferenciada de las funciones ejecutivas que según algunos autores los efectos de la Actividad Física puntual tienen un carácter reversible a corto plazo sobre el sistema cognitivo (McMorris et al., 2009; Tomporowski, 2009).

Veamos, por tanto, los distintos procesos implicados en esta actividad psicológica. La atención selectiva puede definirse como la capacidad para centrarse en uno o dos estímulos importantes, mientras se suprime deliberadamente la consciencia de otros estímulos distractores (Zillmer y Spiers, 1998). Se encarga de controlar los procesos y mecanismos por los cuales el organismo procesa tan sólo una parte de toda la información, y da respuestas tan sólo a aquellas demandas del ambiente que son realmente útiles o importantes para el individuo. En este sentido, la atención selectiva implica la selección de los estímulos que se presentan en el ambiente, y la selección del proceso y/o respuesta que se va a realizar. La atención dividida hace referencia a la actividad mediante la cual se ponen en marcha los mecanismos que el organismo utiliza para dar respuesta ante las múltiples demandas del ambiente. En este caso se trata no de seleccionar aspectos específicos de la información, sino de atender a todo lo que se pueda al mismo tiempo. Y la atención sostenida ha sido definida como la actividad que pone en marcha los procesos y/o mecanismos por los cuales el organismo es capaz de mantener el 
foco atencional y permanecer alerta ante la presencia de determinados estímulos durante períodos de tiempo relativamente largos (Parasuraman, 1984).

Todos estos aspectos diferentes de la atención están regulados por estructuras cerebrales distintas que tienen periodos de neurodesarrollo diferentes (Rebollo y Montiel, 2006). Así, por ejemplo, como han señalado estos mismos autores la atención más elemental o involuntaria depende del funcionamiento del Sistema Reticular Ascendente (SAR) proyectado en toda la corteza cerebral desde el tronco cerebral, de naturaleza noradrenérgica y que se desarrolla precozmente en el primer año de vida.

Si bien hemos descrito que todos estos aspectos diferentes de la atención se vinculan a determinadas estructuras cerebrales que se desarrollan en diferentes momentos de la vida, existe un debate acerca de si se puede hablar o no de un desarrollo atencional en términos estrictos (intradominio), o por el contrario de un desarrollo cognitivo general (interdominio), dentro del cual estaría como un componente más la atención (De Vega y Vega, 1984). García (2008) nos ofrece una revisión de estas teorías agrupándolas del siguiente modo. Por un lado, tendríamos aquellas teorías que consideran que existe un desarrollo progresivo de los distintos mecanismos atencionales que es independiente del desarrollo de los restantes procesos psicológicos.

En este caso se considera que durante la infancia existe una mayor tendencia a la distracción y un menor control atencional en comparación a la vida adulta. Un segundo grupo parte de que el desarrollo atencional depende del desarrollo de otros procesos cognitivos, y en este sentido se acentúa el vínculo existente entre percepción y atención y se considera que ésta es una propiedad selectiva de la percepción. Y, por último, un tercer grupo de teorías sostienen que el desarrollo de la atención va unido al desarrollo cognitivo general, es decir, al desarrollo de otros procesos psicológicos, en este caso no solo a la percepción sino también a la memoria, el pensamiento y a la inteligencia. Solo se considera que el control atencional se desarrolla de forma más específica e independiente de los restantes procesos cognitivos. Por tanto, el rendimiento obtenido por los niños en las pruebas de atención se entiende que depende tanto del desarrollo cognitivo alcanzado como por el nivel de desarrollo del autocontrol atencional.

La capacidad para mantener la atención durante un periodo prolongado de tiempo y la habilidad para responder apropiadamente a estímulos infrecuentes se conoce como atención sostenida o vigilancia (Gunzelmann et al., 2011). Debemos destacar el papel fundamental de la vigilancia durante las tareas que realizamos y el rendimiento en diferentes actividades como el deporte y entornos de trabajo (e.g., controladores aéreos, conductores profesionales, actividades industriales, etc.). Así, un estado de vigilancia óptimo minimiza los errores humanos (Caldwell, Caldwell, Smith y Brown, 2004) 
En el presente estudio hemos abordado un doble objetivo. Por una parte, analizar el desarrollo evolutivo de la atención en alumnos de karate a partir de las medidas y parámetros que establece el Test de atención d2, y por otra, obtener datos normativos para los dichos alumnos de cada uno de los parámetros que se incluyen en esta prueba.

\section{MATERIAL Y MÉTODOS}

\section{Participantes}

La muestra evaluada fueron sujetos escolares que practican Karate y tenían experiencia previa en competición. Participaron 14 Karatekas ( 8 chicas) de Málaga con una edad promedio de $11.32 \pm .63$ años y experiencia similar en el deporte.

Todos los sujetos eran estudiantes de primaria y practicaban el deporte en el mismo club desde su inicio.

\section{Materiales}

Los materiales empleados para el registro psicofisiológico fueron: Un cronómetro, hojas de registro, ordenador portátil y el Test de Atención d2 de atención selectiva (Brickenkamp, 2004).

El test de atención d2 se utiliza para evaluar la atención selectiva y la concentración mediante una prueba de cancelación y mide la velocidad de procesamiento, en una tarea de discriminación de estímulos visuales similares. El test consiste en discriminar entre 47 caracteres que componen una fila, con 14 filas y un total 658 elementos a discriminar. Se dispone de 20" para rellenar cada fila.

\section{Procedimiento}

Para la recogida de datos se realizó en un club de la capital malagueña, previamente se solicitó autorización paterna con un formulario de consentimiento informado, posteriormente se procedió a la realización los test del d 2 previos a una sesión de entrenamiento de Karate. Se le volvió a pasar el instrumento al terminar la sesión.

La división para el posterior análisis se realizó por grupos y por sexo para analizar los cambios producidos.

\section{Análisis de los resultados}

El análisis de los resultados se realizo por medio del programa estadístico SPSS 25 para Mac. Se produjo dos tipos de análisis de los datos, por un lado, descriptivos, y por otro 
inferenciales. A su vez se hizo la prueba de Shapiro-Wilk para comprobar la normalidad de la muestra ya que la muestra es inferior a 30 sujetos.

Para evaluar las diferencias en las puntuaciones entre los grupos se implementó análisis de la varianza (ANOVA). Para analizar las correlaciones entre las medidas objeto de estudio se utilizó el coeficiente bivariado de Pearson al ser una muestra normal.

\section{RESULTADOS}

Los resultados muestran que la distribución de los datos la escala sigue una tendencia normal en todos los casos salvo para las puntuaciones de $\mathrm{C}$.

En cuanto a la prueba del análisis de la varianza (ANOVA) existen diferencias entre grupos, siendo un poco más altas en el grupo de las chicas, aunque estas diferencias no son estadísticamente significativas.

También observamos que una tendencia hacia el aumento de la atención después de realizar el entrenamiento los Karatecas, pero las diferencias no son significativas $(p=.36)$. En el análisis por sexos aparecen resultados similares algo por encima en el grupo de las chicas, pero los aumentos no son significativos $(\mathrm{p}=.43$ chicos, $\mathrm{y} p=.28$ chicas $)$

Como hemos observado todos los deportistas mostraron una mejoría en los resultados de atención mostrando una tendencia aunque esta no sea estadísticamente significativa.

\section{DISCUSION Y CONCLUSIONES}

En el presente estudio hemos analizado el nivel de atención en alumnos de 11 y 12 años, tras la realización de una práctica deportiva, siguiendo los criterios y pautas del test D2 para poder analizar la atención selectiva y la concentración.

Si comparamos nuestros resultados con respecto a la utilización de este test en la educación en la que se han realizado diversos estudios que han analizado el nivel de atención de alumnos en educación primaria y la evolución a través de los años (Jiménez et. Al, 2012) o bien la correlaciones existentes entre atención plena y componentes atencionales (AlbertínMarco, 2012), nos dan resultados similares pero sin significatividad.

Sin embargo, los estudios realizados en relación a la actividad física, son minoritarios. En Jaén se llevó a cabo un estudio con 158 participantes, medido con el test D2, con el fin de conocer el efecto inmediato y en días posteriores, de un programa de 20 minutos de Actividad física sobre la atención y concentración en adolescentes; el resultado obtenido fue una mejora significativa de las mismas tras la realización de AF (González-González, 2017), tal como ocurre en los resultados de nuestro estudio. 
En definitiva, tras la realización del test, hemos observado que la atención de los alumnos incrementa tras la realización de una clase de actividad física, lo cual podemos comprobarlo en el incremento del número total de aciertos (TA) y la disminución de respuestas omitidas por parte del alumnado.

Comparando nuestros resultados con los obtenidos en estos estudios previamente citados y con el reciente artículo de Pérez-Lobato, Reigal y Hernandez-Mendo (2016) podríamos aventuramos a decir que los resultados serían similares, en cuanto a significatividad, si la muestra fuera mayor.

Aún así la tendencia es positiva ya que en el pos test tiene mejores resultados que en el pre test y que en cuanto a género los resultados también son mejores aunque sin ser significativos en las chicas que en los chicos al igual que los estudios previos.

\section{PROSPECTIVA DE FUTURO}

Para poder comprobar y verificar los resultados obtenidos por estudios previos sería necesario realizar este mismo estudio con sujetos escolares pero con una muestra mayor donde los resultados obtenidos tendrían más posibilidades de ser estadísticamente significativos.

En futuros estudios deberíamos intentar tener un grupo de control con alumnos de secundaría que no sean deportistas y otro grupo de deportistas para verificar diferencias entre deportistas y no deportistas en cuanto a atención y así mismo poder realizar una intervención deportiva para poder estudiar los resultados en cuanto a atención.

Hoy día hay números estudios sobre atención y en concreto en atención sostenida que correlacionan un mayor nivel de atención con una mejora en los resultados tanto físicos como psíquicos lo que es interesante seguir investigando y poder utilizar para posteriores investigaciones pruebas de atención sostenida como las PVT (Psycomotor Vigilance Task) muy efectivas y utilizadas en estudios científicos actuales.

\section{Referencias}

Albertín-Marco, R. (2012). Estudio preliminar de correlación entre atención plena y componentes atencionales cognitivos en educación primaria. (Trabajo fin de grado, UNIR) recuperado https://reunir.unir.net/bitstream/handle/123456789/231/Albertin_Raquel.pdf?sequence=1\&isAllowed=y

Brickenkamp, R. (2004). d2: Test de atención-Manual (adapt. Nicolás Seisdedos Cubero; Revisada y Ampliada). Madrid: TEA Ed.

Castillo, M.D. (2009). La atención. Madrid: Pirámide.

Caldwell, J. A., Caldwell, J. L., Smith, J. K. y Brown, D. L. (2004). Modafinil's effects on simulator performance and mood in pilots during $37 \mathrm{~h}$ without sleep. Aviation Space and Environmental Medicine. 
Cuesta, M., de Iscar, M.J., Begega, M.A., Méndez, M., Álvarez, L., Solís, G.,..y Arias, J.L. (2007). Psychometric properties of the $\mathrm{d} 2$ selective attention test in a sample of premature and born-at-term babies. Psicothema, 19, 706-710.

De Vega, M., y Vega, M. (1984). La Atención. Introducción a la Psicología Cognitiva, 123-171.

García, J. (2008). Psicología de la atención. Madrid: Síntesis.

González-González, B. (2017). El efecto semanal de actividad física de alta intensidad en la atención, concentración y memoria en adolescentes. (Trabajo Fin de Máster, Universidad de Jaén). Recuperado de http://tauja.ujaen.es/handle/10953.1/4682.

Gunzelmann, G., Moore, L. R., Gluck, K. A., Van Dongen, H. P. A., y Dinges, D. F. (2011). Fatigue in sustained attention: Generalizing mechanisms for time awake to time on task. Cognitive Fatigue: Multidisciplinary Perspectives on Current Research and Future Applications., 83-101. doi: 10.1037/12343-004

Jiménez, J. E., Hernández, S., García, E., Díaz, A., Rodríguez, C., y Martín, R. (2012). Test de atención D2: Datos normativos y desarrollo evolutivo de la atención en educación primaria. European journal of education and psychology, $5(1)$.

McMorris, T., Davranche, K., Jones, G., Hall, B., Corbett, J., y Minter, C. (2009). Acute incremental exercise, performance of a central executive task, and sympathoadrenal system and hypothalamic-pituitary-adrenal axis activity. International Journal of Psychophysiology, 73(3), 334-340. doi: 10.1016/j.ijpsycho.2009.05.004

Miranda, A., García, R. y Soriano, M. (2005). Habilidad narrativa de los niños con trastorno por déficit de atención con hiperactividad. Psicothema, 17, 227-232.

Parasuranam, R. (1984). Sustained attention in detection and discrimination. En R. Parasuranam y D.R. Davies (Eds.), Varieties of Attention (pp. 243-271). Nueva York: Academic Press.

Pérez-Lobato, R., Reigal, R., y Hernández Mendo, A. (2016). Relaciones entre la práctica física, condición física y atención en una muestra adolescente. Revista de psicología del deporte, 25(1), 0179-186.

Rebollo, M.A. y Montiel, S. (2006). Atención y funciones ejecutivas. Revista de Neurología, 42, 3-7.

Tomporowski, P. D. (2009). Methodological Issues: Research Approaches, Research Design, and Task Selection. In Exercise and Cognitive Function. doi: 10.1002/9780470740668.ch4

Vega, M. (1984). Introducción a la psicología cognitiva. Madrid: Alianza Editorial.

Zillmer, E.A. y Spiers, M.V. (1998). Principles of clinical neuropsychology. Pacific Groove, CA: Brooks/Cole. 